\section{Insatisfação corporal, prática de dietas e comportamentos de risco para transtornos alimentares em mães residentes em Santos}

\author{
Body dissatisfaction, dieting and risk behaviors for eating \\ disorders among mothers living in Santos
}

Fernanda Baeza Scagliusi', Patrícia da Rocha Pereira², Isis de Carvalho Stelmo², Ramiro Fernandez Unsain³, Paula Andrea Martins', Priscila de Morais Sato?

\section{RESUMO}

Objetivo: Avaliar a insatisfação corporal, a prática de dietas e os comportamentos de risco para transtornos alimentares em uma amostra de mães residentes no município de Santos. Métodos: Foi realizado um estudo transversal, de base populacional, com 453 mães de filhos com até 10 anos de idade. As mães responderam ao Teste de Atitudes Alimentares (EAT-26), à Escala de Figuras de Stunkard e a uma questão sobre a prática atual de dietas. Resultados: Das mães, 29,9\% apresentaram escore positivo para os comportamentos de risco para transtornos alimentares e 21,8\% estavam fazendo dieta para emagrecer no momento da entrevista. No tocante à imagem corporal, 17,5\% das mães estavam satisfeitas com o seu tamanho corporal, 71,5\% gostariam de diminuir seu tamanho corporal e 11,0\% gostariam de aumentá-lo. Os comportamentos de risco para transtornos alimentares foram mais frequentes nas mães insatisfeitas com seus tamanhos corporais ( $p<0,0001)$. Conclusão: A maioria das mães investigadas estava insatisfeita com os seus tamanhos corporais. A frequência de mães que praticavam dietas ou tinham comportamentos de risco para transtornos alimentares foi similar ou superior aos demais estudos nacionais, conduzidos, em sua maioria, com populações consideradas de risco, como meninas adolescentes e jovens universitárias.

\begin{abstract}
Objective: To evaluate body dissatisfaction, dieting and risk behaviors for eating disorders among a sample of mothers living in the city of Santos. Methods: It was conducted a cross-sectional population-based study with 453 mothers of children aged until 10 years old. The mothers answered to the Eating Attitudes Test (EAT-26), the Stunkard's Figure Scale and a question about being currently dieting. Results: $29.9 \%$ presented a positive score for risk behaviors for eating disorders and $21.8 \%$ were currently dieting. Regarding body image, $17.5 \%$ of the mothers were satisfied with their current body size, $71.5 \%$ would like to decrease their body sizes and $11.0 \%$ would like to increase it. The risk behaviors for eating disorders were more frequent among the body-dissatisfied mothers $(p<0.0001)$. Conclusion: Most of the mothers were dissatisfied with their body sizes. The frequency of mothers who practiced
\end{abstract}

1 Universidade de São Paulo (USP), Universidade Federal de São Paulo (Unifesp), Campus Baixada Santista, Instituto de Saúde e Sociedade, Departamento de Saúde, Clínica e Instituições.

2 Unifesp, Campus Baixada Santista, Instituto de Saúde e Sociedade, Departamento de Saúde, Clínica e Instituições.

3 Universidad de Buenos Aires, Faculdad de Filosofía y Letras.

4 Unifesp, Campus Baixada Santista, Instituto de Saúde e Sociedade, Departamento de Ciências do Movimento Humano.

Endereco para correspondência: Fernanda Baeza Scagliusi

Universidade Federal de São Paulo, Campus Baixada Santista, Instituto de Saúde e Sociedade, Departamento de Saúde, Clínica e Instituições Rua Silva Jardim, 136, Vila Mathias

$11015-020$ - Santos, SP

Telefax: (13) 3878-3700

E-mail: fernanda.scagliusi@gmail.com 


\section{Keywords}

Eating disorders, diet, body

image, mothers. diets or presented risk behaviors for eating disorders was similar or superior to the rest of the national studies, conducted, at large, with populations considered at risk, such as adolescent girls or young college women.

\section{INTRODUÇÃO}

A alimentação e o corpo são objetos epistêmicos complexos, com dimensões multifacetadas e inserções contextuais profundas', que se mostram pungentes na contemporaneidade'. Segundo Santos', a "relação temática entre o corpo e o comer, embora possa parecer óbvia em uma primeira vista, concebe a comida como construtora do corpo não só do ponto de vista da sua materialidade como também nos seus aspectos socioculturais e simbólicos".

O contexto de modernidade alimentar foi apontado por Fischler ${ }^{3}$ como uma "gastroanomia", caracterizada pela abundância alimentar, pela diminuição dos controles sociais sob a alimentação e pela multiplicação dos discursos sobre o que é necessário ingerir. Tais discursos englobam as normas dietéticas, com suas variações e contradições, os ideais estéticos de magreza e a popularização e a vulgarização das estratégias para atingir tal ideal, das quais se destaca a dieta. Fischler ${ }^{3}$ também afirmou que "é na brecha da anomia que proliferam as pressões múltiplas e contraditórias que se exercem sobre o comedor moderno. [...] A liberdade anômica é também um conflito ansioso, e esta ansiedade sobredetermina, por sua vez, as condições alimentares aberrantes".

Como sintomas dessa conjuntura, são observados os fenômenos de práticas de dietas e de insatisfação corporal reconhecidos como fatores de risco para os transtornos alimentares ${ }^{4}$. Os transtornos alimentares são quadros psiquiátricos de etiologia multifatorial, caracterizados por alterações nas práticas alimentares e por distúrbios de imagem corporal $^{4}$. O diagnóstico dos transtornos alimentares deve ser feito por um psiquiatra, que pode utilizar, para tanto, entrevistas estruturadas. Em estudos científicos, especialmente naqueles com grandes amostras, a realização de entrevistas conduzidas por psiquiatras, com fins diagnósticos, acarreta maiores custos, uma vez que essa demanda maior tempo para avaliação e entrevistadores treinados 5 . Instrumentos de avaliação, como escalas e questionários, apresentam aplicação rápida e econômica e avaliam sintomas de transtornos alimentares em uma dada população, sem, entretanto, realizar um diagnóstico". Tais sintomas podem ser chamados de "comportamentos de risco para transtornos alimentares", uma vez que precedem os quadros clínicos clássicos ${ }^{6}$. Estudos que avaliem a ocorrência de tais fenômenos em diferentes populações são importantes para identificar quais grupos requerem esforços intensificados de prevenção e tratamento.

No Brasil, diversos estudos avaliaram a ocorrência da insatisfação corporal, prática de dietas e comportamentos de risco para transtornos alimentares ${ }^{7-43}$. Contudo, a amostra da maioria deles foi composta por crianças e adolescentes ${ }^{8-23}$ ou por estudantes universitários ${ }^{24-37}$. Apenas um estudo avaliou gestantes ${ }^{41}$, porém, até o limite do nosso conhecimento, nenhum estudo nacional de base populacional avaliou tais fenômenos especificamente entre mães que não estejam grávidas. Destaca-se que as mães têm um ganho de peso importante durante a gestação e que, geralmente, esse peso adquirido dificilmente é perdido após a gestação ${ }^{44}$. Sabe-se que ganho de peso é fator de risco para o desenvolvimento de obesidade e transtornos alimentares ${ }^{45}$. Assim, é possível que a população de mães seja um grupo de risco para transtornos alimentares. Dessa forma, o presente estudo teve como objetivo avaliar a insatisfação corporal, a prática de dietas e os comportamentos de risco para transtornos alimentares em uma amostra de mães residentes no município de Santos (São Paulo).

\section{MÉTODOS}

\section{Delineamento do estudo e da amostragem}

O presente estudo está inserido em duas pesquisas em andamento: "Avaliação do ambiente nutricional do município de Santos" e "Influência das práticas alimentares e do ambiente nutricional sobre o ganho de peso em mães residentes no município de Santos". A coleta de dados foi realizada entre janeiro de 2010 e fevereiro de 2011.

Foi realizado um estudo transversal de base populacional na cidade de Santos, com mães de crianças de até 10 anos de idade. Os critérios de exclusão foram: a) estar grávida; b) estar com câncer; c) apresentar AIDS ou HIV positivo; d) ter realizado cirurgia bariátrica; e e) residir em Santos apenas na temporada de veraneio. Ressalta-se, porém, que não foram feitos exames para constatar os quatro primeiros itens; eles foram obtidos pelo relato.

Utilizou-se o plano complexo de amostragem por conglomerados, tendo como unidades primárias os setores censitários do Censo de 2000 do IBGE. O município de Santos possui cinco regiões (Centro, Noroeste, Orla, Porto e Morros), as quais possuem 533 setores censitários na região insular, com 130.478 domicílios. Por causa da violência e da dificuldade de acesso, foram excluídas as regiões dos Morros e do Porto, já que contavam com um número pequeno de domicílios (respectivamente, 7,3\% e 0,7\% do total). Foi feita a estratificação implícita por região e renda de conglomerados, com partilha proporcional ao número de domicílios particulares permanentes, resultando no sorteio de 35 setores 
censitários. As três regiões estudadas (Orla, Centro e Zona Noroeste) diferem quanto ao nível socioeconômico, e a Orla possui maior nível.

Após o sorteio, os setores foram percorridos, para identificação dos domicílios elegíveis. Foram feitas três tentativas de realização da entrevista no domicílio, sendo uma de fim de semana quando necessário. Após a identificação de domicílios elegíveis, propôs-se a participação no estudo, sendo realizada a entrevista após a assinatura do Termo de Consentimento Livre e Esclarecido.

De acordo com o delineamento amostral inicialmente planejado, seriam sorteadas 16 famílias em cada setor entre as identificadas como elegíveis. No entanto, em virtude de os critérios de exclusão limitarem esse número e pelas dificuldades do trabalho de campo, foram avaliadas todas as mães identificadas nos setores censitários que aceitassem participar do estudo, até serem obtidas, no máximo, 16 entrevistas por setor. Tais cálculos visavam obter a amostra necessária para as pesquisas supracitadas, que abrigam o presente estudo. O n amostral foi de 453 mães, e a taxa de resposta foi de $80,9 \%$.

Cada mãe foi entrevistada duas vezes, com intervalo de 15 a 90 dias. Na primeira entrevista, foram coletadas informações demográficas e socioeconômicas, que são utilizadas no presente trabalho para caracterizar a amostra. Na segunda entrevista, foram aplicados os instrumentos de interesse para o presente estudo, que compreendiam questões sobre imagem corporal, práticas de dietas e comportamentos de risco para transtornos alimentares. As entrevistas foram realizadas por entrevistadores treinados, com duração de $30 \pm$ 10 minutos.

O estudo foi aprovado pelo Comitê de Ética em Pesquisa da Universidade Federal de São Paulo (Protocolo n 0300/10).

\section{Instrumentos de avaliação}

Os comportamentos de risco para transtornos alimentares foram avaliados pelo Eating Attitude Test $\left(\right.$ EAT-26) ${ }^{46}$. Ele possui 26 questões em escala Likert, que indagam sobre sintomas de restrição alimentar, comportamentos anoréxicos e preocupações anormais sobre alimentação e imagem corporal. Cada pergunta apresenta seis opções de resposta, sendo três positivas (sempre, muitas vezes e às vezes) e três negativas (nunca, quase nunca e poucas vezes). O EAT-26 é utilizado como um instrumento de rastreamento de sintomas de transtorno alimentar, sem fazer um diagnóstico de tal quadro. A pontuação máxima que pode ser obtida é de 78 pontos (e a mínima é de zero ponto), e escores maiores ou iguais a 21 pontos sugerem comportamentos de risco para transtornos alimentares. Foi utilizada a versão validada no Brasil por Bighetti et al. ${ }^{47}$.

O próprio EAT-26 apresenta uma questão sobre a prática de dietas, formulada da seguinte maneira: "Você faz regi- mes para emagrecer?". Como tal questão não especifica o período de tempo ao qual se refere, as respostas dadas a ela foram interpretadas no presente estudo como um indicativo da prática habitual de dietas. Além disso, os sujeitos foram questionados se estavam fazendo dieta ou regime para emagrecimento no período da entrevista.

A insatisfação corporal foi analisada a partir da Escala de Figuras de Stunkard ${ }^{48}$, que consta de nove figuras que variam de magras a obesas. Essa escala foi validada para mulheres brasileiras por Scagliusi et al. ${ }^{49}$. O sujeito escolhia uma figura que representasse como ele se vê atualmente, uma segunda figura que representasse o tamanho corporal considerado por ele saudável e uma terceira figura que representasse o tamanho corporal que ele julga ideal.

Cada figura recebe um escore que varia de 1 para a mais magra a 9 para a mais obesa. Foi calculado o escore da discrepância entre o tamanho atual e o ideal - que corresponde ao escore da figura atual menos o escore da figura ideal. Tal escore foi utilizado como uma medida de insatisfação corporal.

\section{Análise estatística}

As análises foram conduzidas com o auxílio do software SPSS versão 19, no módulo de análises complexas, considerando-se os pesos amostrais.

Em relação ao EAT-26, foram analisados o escore obtido (como média, erro-padrão, valores mínimos e máximos) e a frequência de mães que apresentaram comportamentos de risco para transtornos alimentares (aquelas cujos escores do EAT-26 foram $\geq 21$ pontos). Ademais, foi analisada a frequência de respostas positivas (isto é: sempre, muitas vezes e às vezes) para as seguintes perguntas desse teste: "Você faz regimes para emagrecer?" e "Você provoca vômitos depois de comer?".

Ainda no tocante à prática de dietas, foi observada a frequência de mães que responderam afirmativamente à questão: "Você está fazendo dieta ou regime para emagrecer?".

Em relação à imagem corporal, foi calculado o escore de insatisfação corporal (discrepância entre o tamanho atual e o ideal) e este é apresentado como média, erro-padrão, mediana, valores mínimos e máximos. Tal escore pode variar entre -8 e 8 . Assim, foram analisadas as frequências de mães que: 1) estavam satisfeitas com o seu tamanho corporal (cujos escores de discrepância eram iguais a zero); 2) gostariam de diminuir seu tamanho corporal (cujos escores eram positivos); e 3) gostariam de aumentar seu tamanho corporal (cujos escores eram negativos). Ademais, foram observadas as distribuições das figuras escolhidas como atual, saudável e ideal.

O teste do qui-quadrado foi utilizado para comparar a proporção de sujeitos com e sem comportamentos de risco para transtornos alimentares entre as categorias supracitadas (participantes que gostariam de aumentar, manter ou diminuir seus tamanhos corporais). 


\section{RESULTADOS}

Foram analisadas 453 mulheres; dessas, 36,6\% residiam na região do Centro, 39,6\%, na Orla e 23,8\%, na Zona Noroeste. As idades variaram de 17 a 55 anos, sendo a idade média e seu erro-padrão, respectivamente, 33,2 e 0,35 anos. A população estudada era majoritariamente branca $(70,3 \%)$, sendo $19,7 \%$ de negras ou mulatas, $0,5 \%$ de orientais e $0,4 \%$ de indígenas (9,1\% da amostra não apresentaram resposta para essa variável). Com relação à escolaridade, 13,1\% não tinham instrução ou apresentavam ensino fundamental incompleto; $5,9 \%$ possuíam ensino fundamental completo; $8,6 \%$ apresentavam ensino médio incompleto; $42,6 \%$ tinham ensino médio completo; 8,0\% possuíam ensino superior incompleto e $21,8 \%$ apresentavam ensino superior completo.

O escore médio do EAT-26 foi de 17,0 pontos, com erro-padrão de 0,44 pontos. O escore mínimo obtido foi de 1 ponto, enquanto o máximo foi de 48 pontos. A proporção de mães que apresentaram escore positivo para os comportamentos de risco para transtornos alimentares foi de $29,9 \%$.
Por sua vez, 2,4\% das mães apresentaram respostas positivas para a questão "Você provoca vômitos depois de comer?". A pergunta "Você faz regimes para emagrecer?" foi respondida positivamente por 38,6\% das mães. Ademais, 21,8\% relataram estar fazendo dieta para emagrecer no momento presente da entrevista.

As estatísticas descritivas referentes à Escala de Figuras de Stunkard encontram-se na tabela 1. A figura 1 apresenta as distribuições das figuras escolhidas como atuais, saudáveis e ideais. Percebe-se que a maior parte das mães escolheu figuras entre a 3 e a 6 para representar seu tamanho atual, enquanto as figuras 2 a 4 foram mais escolhidas como tamanhos saudáveis e ideais. Segundo o escore de discrepância entre as figuras atuais e ideais, $17,5 \%$ das mães estavam satisfeitas com o seu tamanho corporal, 71,5\% gostariam de diminuir seu tamanho corporal e 11,0\% gostariam de aumentá-lo. A tabela 2 compara a proporção de mães com e sem comportamentos de risco para transtornos alimentares entre as categorias supracitadas de escore de discrepância entre as figuras atuais e ideais.

Tabela 1. Estatísticas descritivas das medidas de imagem corporal obtidas pela Escala de Figuras de Stunkard, aplicada a 453 mães residentes no município de Santos

\begin{tabular}{lcccc}
\hline & Figura atual & Figura saudável & Figura ideal & Escore de insatisfaçäo corporal* $^{*}$ \\
\hline Média & 4,5 & 3,1 & 3,2 & 1,3 \\
Erro-padrão & 0,08 & 0,05 & 0,05 & 0,08 \\
Mediana & 4,0 & 3,0 & 3,0 & 1,0 \\
Valor mínimo & 1,0 & 1,0 & 1,0 & $-6,0$ \\
Valor máximo & 9,0 & 8,0 & 8,0 & 8,0 \\
\hline
\end{tabular}

* Calculado como: escore da figura atual - escore da figura ideal.

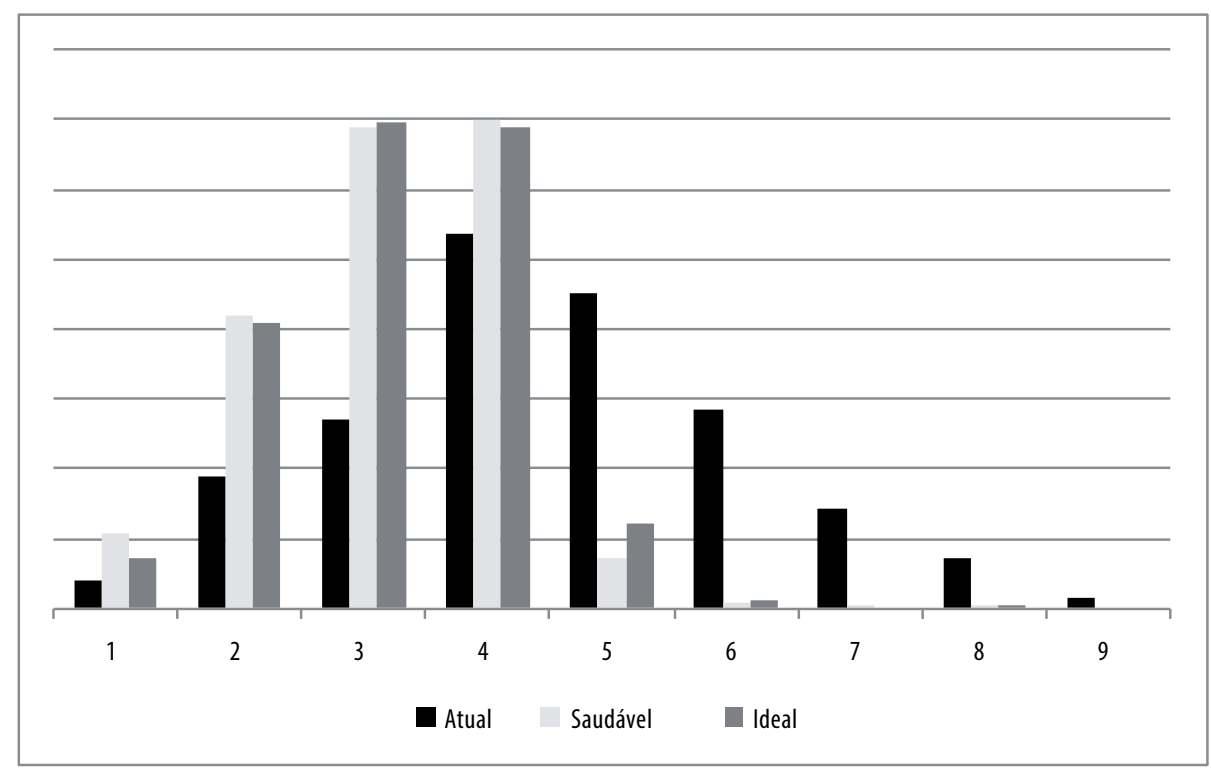

Figura 1. Distribuições das figuras escolhidas como atuais, saudáveis e ideais, obtidas pela Escala de Figuras de Stunkard, aplicada a 453 mães residentes no município de Santos. 
Tabela 2. Proporção de mães residentes no município de Santos ( $n=453)$ com comportamentos de risco para transtornos alimentares (avaliados pelo teste EAT-26), de acordo com o sentido dos escores de discrepância entre o tamanho corporal atual e o ideal (avaliados pela Escala de Figuras de Stunkard)*

\begin{tabular}{lcc}
\hline & $\begin{array}{c}\text { Proporçäo de mães sem comportamentos de risco para transtornos } \\
\text { alimentares }\end{array}$ & $\begin{array}{c}\text { Proporçäo de mães com comportamentos de risco para transtornos } \\
\text { alimentares }\end{array}$ \\
\hline Sujeito deseja aumentar seu tamanho corporal & $81,4 \%$ & $18,6 \%$ \\
Sujeito está satisfeito com o seu tamanho corporal & & $8,3 \%$ \\
Sujeito deseja diminuir seu tamanho corporalc & $91,7 \%$ & $37 \%$ \\
\hline
\end{tabular}

${ }^{*} \times 2(2)=28,4 ; p<0,0001$

a Sujeitos cujos escores de discrepância entre o tamanho atual e o ideal eram negativos.

' Sujeitos cujos escores de discrepância entre o tamanho atual e o ideal eram igual a zero.

' Sujeitos cujos escores de discrepância entre 0 tamanho atual e 0 ideal eram positivos.

\section{DISCUSSÃO}

O presente estudo encontrou, nessa amostra de mães do município de Santos, uma ocorrência de comportamentos de risco para transtornos alimentares e práticas de dietas similar a de outras populações brasileiras. Ademais, a maioria das mães apresentava insatisfação corporal. O EAT-26, instrumento utilizado na presente pesquisa para avaliar os comportamentos de risco para transtornos alimentares, serve como uma ferramenta de rastreamento de sintomas. Não é possível realizar afirmações sobre a prevalência dos quadros clínicos de transtornos alimentares nessa amostra, porém é possível sinalizar uma situação de vulnerabilidade. Provavelmente, o termo mais adequado para designar esses comportamentos de risco seja o "comer transtornado", definido como comportamentos alimentares restritivos e disfuncionais e métodos inadequados para perder ou controlar o peso corporal, que ocorrem com menor frequência e de forma menos grave do que o exigido pelos critérios diagnósticos de transtornos alimentares ${ }^{50}$. Sabe-se que o comer transtornado está associado à insatisfação corporal ${ }^{51}$, de tal forma que ambos formam um fenômeno ou quadro complexo. Essa afirmação é corroborada pelos dados do presente estudo, uma vez que a frequência de comportamentos de risco para transtornos alimentares foi maior entre as mães que estavam insatisfeitas com seus tamanhos corporais e, principalmente, entre aquelas que desejavam diminuí-los.

Os estudos acerca desses fenômenos têm se concentrado mais em amostras de adolescentes e mulheres jovens, especialmente universitárias, justamente por esses serem considerados grupos de maior risco ${ }^{51,52}$. A literatura sobre tais fenômenos no grupo materno se debruça mais sobre as seguintes linhas de pesquisa: 1) caracterização das mães cujos filhos apresentam transtornos alimentares ${ }^{40}$; 2 ) análise do efeito das características maternas sobre aspectos alimentares e corporais dos filhos ${ }^{53}$; e 3) descrição dos riscos e prognósticos de mulheres com transtorno alimentar que engravidam ${ }^{53}$. Sugere-se ampliar o foco das pesquisas, para que os comportamentos de comer transtornado e a imagem corporal sejam in- vestigados no grupo materno, especialmente em estudos de base populacional, já que a insatisfação corporal parece ser estável ao longo da vida ${ }^{51}$ e não melhorar com a maternidade ${ }^{54}$.

Até o limite do nosso conhecimento, 23 estudos nacionais utilizaram o EAT-26 para rastrear comportamentos de risco para transtornos alimentares. Desses, um avaliou muIheres de 12 a 29 anos (com uma frequência de 16,5\% de escore positivo) ${ }^{7}$, um investigou mães de filhas com e sem transtornos alimentares ${ }^{40}, 12$ analisaram amostras de universitárias ${ }^{24-35}$, seis avaliaram crianças e adolescentes $8,10,12,13,15,22$ e três analisaram atletas ${ }^{38,39,43}$. A frequência de escore positivo encontrada no presente estudo é superior à da maioria dos estudos publicados até então. Maiores frequências foram observadas apenas entre as adolescentes estudantes de escolas particulares de Dunker et al. ${ }^{12}$, com 34,3\% de escore positivo, e entre as estudantes de Nutrição de Antonac$\mathrm{cio}^{24}$ e Laus et al. ${ }^{33}$, com, respectivamente, $40 \%$ e $50 \%$ de escore positivo. No estudo com mães de filhas com e sem transtornos alimentares, a frequência de escore positivo variou entre $4,8 \%$ e $8,6 \%$, porém seu tamanho amostral ( $n=$ 70) e o fato de o grupo controle ter sido formado apenas por mães cujas filhas estudavam em uma escola particular de São Paulo limitam sua abrangência e comparabilidade ${ }^{40}$. Dessa forma, embora a maior parte da amostra investigada no presente estudo não apresente comportamentos de risco para transtornos alimentares, nota-se que a frequência de mães com tais comportamentos é similar ou superior à dos demais estudos supracitados, conduzidos, majoritariamente, com populações consideradas de risco, como meninas adolescentes e universitárias. Ressalta-se, todavia, que poucas mães relataram praticar vômitos autoinduzidos, que seria um comportamento mais típico dos quadros clínicos de transtornos alimentares. Usando o Eating Disorder Examination para a investigação de gestantes atendidas na atenção básica à saúde $(n=515)$, Soares et al. ${ }^{41}$ encontraram que $0,8 \%$ das gestantes apresentava um possível diagnóstico de transtornos alimentares. Comparar tal dado com aqueles obtidos no presente estudo é difícil, uma vez que o instrumento aqui empregado (EAT-26) apenas rastreia sintomas, sem indicar possíveis diagnósticos. 
Já os dados nacionais sobre a prática de dietas são escassos, e não foi encontrado nenhum estudo com adultos e/ou mães. O levantamento populacional de Pelotas encontrou que $14,4 \%$ das meninas adolescentes haviam feito dietas nos últimos três meses ${ }^{17}$. Já no trabalho de Ferreira e Veiga ${ }^{11}$, com adolescentes de 12 a 19 anos, 24,7\% faziam dieta rigorosa pelo menos uma vez por semana. Os resultados do presente estudo corroboram tal panorama, mostrando que uma parcela das mães costuma praticar dietas, conforme observado em adultas de outros países, como nos Estados Unidos ${ }^{55}$.

Alguns estudos brasileiros utilizaram a Escala de Figuras de Stunkard para avaliar a insatisfação corporal em diferentes populações. Um recente levantamento populacional conduzido em Florianópolis encontrou que $66 \%$ das adultas analisadas gostariam de diminuir seu tamanho corporal ${ }^{42}$. Já um estudo com universitárias das cinco regiões brasileiras encontrou uma distribuição das figuras escolhidas como atuais, saudáveis e ideais muito semelhante à nossa ${ }^{37}$. Essas comparações reforçam a hipótese de que esta amostra de mães apresenta uma frequência de insatisfação corporal similar à de outros estudos que investigaram populações tipicamente consideradas de risco para distúrbios de imagem corporal, como universitárias $28,30,31,37$.

Os resultados observados pelo nosso estudo podem ser interpretados e problematizados em duas abordagens; uma voltada para as consequências dos fenômenos analisados para a saúde e outra de caráter sociocultural, que inclui perspectivas históricas e de gênero. Para ambas, é interessante a noção de que existe um continuum de questões alimentares e corporais, no qual os transtornos alimentares se situariam no final do espectro. Os fenômenos verificados nesta pesquisa podem corresponder a manifestações parciais dos transtornos, as quais estariam situadas ao longo de tal espectro.

Na perspectiva da saúde, sabe-se que tais manifestações parciais podem evoluir para quadros completos, cujo prognóstico tende a ser ruim e cujo tratamento tende a ser demorado e custoso $0^{56}$. Portanto, a identificação precoce de tais manifestações parciais e a realização de programas de prevenção são necessárias. A insatisfação corporal, além de ser fator de risco para os transtornos alimentares, está associada à depressã $0^{57}$. Alguns autores têm proposto que a insatisfação corporal é um fator de risco compartilhado pela obesidade e pelos transtornos alimentares, embora as evidências para tal hipótese sejam oriundas de estudos com crianças e adolescentes $^{45}$. Já os efeitos da prática de dietas sobre a saúde são controversos. O termo "prática de dietas" é extremamente amplo e vago, podendo abranger comportamentos bastante distintos. Há evidências, embora ainda não consolidadas, de que a prática de dietas é um método ineficaz de controle do peso corporal que pode contribuir para o desenvolvimento de compulsão alimentar e do "efeito sanfona"58.

Em uma perspectiva mais ampla, os resultados do presente trabalho podem levar à reflexão sobre a vivência femi- nina em um contexto sociocultural de hipervalorização do corpo. Os padrões estéticos são construções socioculturais que não podem ser examinadas sem os devidos referenciais temporais e espaciais. Conforme salientado por Scagliusi e Lourenço ${ }^{2}$, a análise dos padrões estéticos deve considerar os antecedentes históricos relacionados ao controle social do corpo feminino dentro das sociedades patriarcais. As autoras afirmam que as intervenções voltadas para a perda de peso reforçaram a cultura da magreza e, com as indústrias de alimentos, cosméticos e roupas, contribuíram para a epidemia de práticas de dietas desnecessárias e perigosas entre as mulheres. Apontam também que o crescimento e o desenvolvimento da mulher no contexto de modernidade representam o ápice da proficiência em dietas. Santos ${ }^{1}$ destaca que a identidade feminina é expressa por meio do que a mulher não come. Vester ${ }^{59}$ revisou a história do advento da dieta na América moderna. Segundo a autora, no final do século XIX, os conselhos para perda de peso focavam o corpo masculino, associando o emagrecimento à influência econômica, ao poder político e aos privilégios sociais. Naquele momento, as dietas representavam um novo sistema de controle corporal, associado ao homem de classes média e alta, baseado na racionalidade e na força de vontade. O ideal de beleza feminino era de formas arredondadas e macias. Nas mulheres, a barriga protuberante denotava fertilidade e a ausência de tônus muscular refletia seu status de "boneca ou brinquedo", numa sociedade tão civilizada que não precisava do seu trabalho e da sua força física. As ativistas dos direitos da mulher encorajavam suas seguidoras a controlar sua ingestão alimentar, argumentando que as mulheres podiam controlar seus corpos também. Em última análise, a autora mostra como esse potencial libertário foi cooptado pelas práticas normativas hegemônicas, de tal forma que, com o direito de controlar seus corpos, as mulheres ganharam também a responsabilidade e a obrigação de fazê-lo. Em suma, tais perspectivas de gênero e seu contexto sociocultural devem ser aprofundados para que os fenômenos encontrados no presente estudo não sejam enquadrados em um modelo teórico apenas médico e/ou psicológico, como alerta Santos'.

O presente estudo apresenta algumas limitações. Sabe-se que a ausência de exames para confirmar os critérios de exclusão é uma limitação, porém esta pesquisa não possuía condições financeiras e logísticas para realizar esses exames.

Outra limitação foi a utilização de uma versão do EAT26 validada para adolescentes do sexo feminino. Existe outra versão do EAT-26 para adultos ${ }^{60}$, entretanto essa obteve menor consistência interna do que a versão aqui utilizada e não realizou algumas avaliações psicométricas, como a equivalência semântica e a análise fatorial.

A exclusão dos domicílios situados nos Morros e no Porto poderia ser considerada uma limitação, conquanto isso acarretou em uma perda de apenas $8 \%$ dos domicílios, que foi 
considerada no plano de amostragem. Alguns domicílios do Porto não constituem, inclusive, residências permanentes. Subsequentemente, a região dos Morros apresenta baixo nível socioeconômico, assim como duas regiões que foram, de fato, incluídas na amostragem (Centro e Zona Noroeste).

Outra limitação é o uso exclusivo da Escala de Figuras de Stunkard para avaliar a insatisfação corporal. Essa escala já foi criticada por causa do pequeno número de figuras disponibilizadas e do fato de que a variação de tamanho não é uniforme entre as figuras ${ }^{61}$. Entretanto, estudos nacionais confirmam as adequadas propriedades psicométricas desse instrumento ${ }^{49}$. Também se pode argumentar que o construto insatisfação corporal é assaz complexo para ser avaliado apenas pela discrepância entre o tamanho corporal atual e o ideal, já que ele também envolve aspectos afetivos. Mesmo compreendendo tal limitação, optou-se pelo uso dessa escala pela sua rápida aplicação e seu fácil entendimento, características desejáveis em estudos populacionais. Contudo, sugere-se que estudos futuros acerca da imagem corporal de mães brasileiras utilizem também instrumentos mais abrangentes, como o Body Shape Questionnaire e o Body Attitudes Questionnaire.

Finalmente, o uso de perguntas fechadas e da análise quantitativa não permite entender como as práticas de dieta e o comer transtornado se operacionalizam no dia a dia. Embora nosso estudo tenha encontrado que essa amostra de mães apresenta ocorrência similar de comer transtornado ao de diversos outros grupos populacionais, não se pode afirmar que os comportamentos são os mesmos. Um estudo qualitativo mostrou que mulheres obesas e pobres, no Brasil, apresentam uma relação complexa com os alimentos, que alterna períodos de restrição (pois, devido à insegurança alimentar, os poucos alimentos disponíveis são destinados prioritariamente aos homens e aos filhos) com períodos de ingestão excessiva de alimentos baratos, calóricos e pouco nutritivos $^{62}$. É possível especular que o comer transtornado apresentado por parte do grupo aqui analisado (provavelmente as mães de menor nível socioeconômico) se assemelhe mais a esse padrão do que aos comportamentos típicos de transtorno alimentar observados em adolescentes. O achado de que mães que queriam aumentar seus tamanhos corporais apresentavam maior frequência de comportamentos de risco para transtornos alimentares do que aquelas que estavam satisfeitas também é de difícil compreensão. Certamente, estudos qualitativos são necessários para que essas questões sejam esclarecidas.

Ressalta-se que o presente trabalho teve caráter descritivo e que são necessárias pesquisas futuras para identificar os fatores associados ao comer transtornado, à prática de dietas e à insatisfação corporal em mães, como, por exemplo, peso corporal, exposição à mídia, nível socioeconômico, raça e ganho de peso gestacional.

\section{CONCLUSÃO}

Há uma lacuna na literatura científica brasileira sobre o comer transtornado, as práticas de dietas e a insatisfação corporal entre mães. O presente estudo contribui para preencher uma parte dessa lacuna, indicando que a maioria das mães investigadas apresentava insatisfação corporal e que a frequência de mães que praticavam dietas ou comportamentos de risco para transtornos alimentares foi similar ou superior aos demais estudos nacionais, conduzidos, em sua maioria, com populações consideradas de risco, como meninas adolescentes e jovens universitárias.

\section{AGRADECIMENTOS}

O estudo foi financiado pelo Conselho Nacional de Desenvolvimento Científico e Tecnológico (CNPq) (processos 501275/2009-6 e 478212/2009-7) e pela Fundação de Amparo à Pesquisa do Estado de São Paulo (Fapesp) (processos 2009/01361-1 e 2010/04255-5).

\section{CONFLITOS DE INTERESSE}

Não há conflitos de interesse envolvidos nesta pesquisa.

\section{REFERÊNCIAS}

1. Santos LAS. Da anorexia à obesidade: considerações sobre o corpo na sociedade contemporânea. In: Diez-Garcia RW, Cervato-Mancuso AM. Mudanças alimentares e educação nutricional. Rio de Janeiro: Guanabara-Koogan; 2011. p. 109-17.

2. Scagliusi FB, Lourenço BH. A ditadura da beleza e suas consequências no discurso nutricional. In: Alvarenga M, Scagliusi FB, Philippi ST. Nutrição e transtornos alimentares: avaliação e tratamento. Barueri, SP: Manole; 2011. p. 59-84.

3. Fischler C. Gastro-nomie et gastro-anomie. Communications. 1979;31:189-210

4. American Psychiatry Association (APA). Diagnostic and Statistical Manual of Mental Disorders (DSM-IV). 4a ed. Washington DC: American Psychiatry Association; 1994.

5. Moya T, Scagliusi FB. Instrumentos de avaliação em transtornos alimentares. In: Alvarenga M, Scagliusi FB, Philippi ST. Nutrição e transtornos alimentares: avaliação e tratamento. Barueri, SP: Manole; 2011; p. 145-72.

6. Freitas S, Gorenstein C, Appolinario JC. Instrumentos para a avaliação dos transtornos alimentares. Rev Bras Psiquiatr. 2002;24:34-8.

7. Nunes MA, Barros FC, Olinto MTA, Camey S, Mari JDJ. Prevalence of abnormal eating behaviors and inappropriate methods for weight control in young women from Brazil: a population-based study. Eat Weight Disord. 2003;8:100-6.

8. Vilella JEM, Lamounier JA, Dellaretti Filho MA, Neto JRB, Horta GM. Transtornos alimentares em escolares. J Pediatr. 2004;80:49-54.

9. Conti MA, Frutuoso MFP, Gambardella AMD. Excesso de peso e insatisfação corporal em adolescentes. Rev Nutr. 2005;18:491-7.

10. Alves E, Vasconcelos FAG, Calvo MCM, Neves J. Prevalência de sintomas de anorexia nervosa e insatisfação com a imagem corporal em adolescentes do sexo feminino do município de Florianópolis, Santa Catarina, Brasil. Cad Saude Publica. 2008;24:503-12.

11. Ferreira JES, Veiga GV. Eating disorder risk behavior in Brazilian adolescents from low socio-economic level. Appetite. 2008:51:249-55. 
12. Dunker KLL, Fernandes CPB, Carreira Filho D. Influência do nível socioeconômico sobre comportamentos de risco para transtornos alimentares em adolescentes. J Bras Psiquiatr. 2009;58:156-61.

13. Sampei MA, Sigulem DM, Novo NF, Juliano Y, Colugnati FAB. Atitudes alimentares e imagem corporal em meninas adolescentes de ascendência nipônica e caucasiana em São Paulo (SP). J Pediatr. 2009;85:122-8.

14. Castro IR, Levy RB, Cardoso LO, Passos MD, Sardinha LM, Tavares LF, et al. Body image, nutritional status and practices for weight control among Brazilian adolescents. Ciênc Saúde Coletiva. 2010;15:3099-108.

15. Scherer FC, Martins CR, Pelegrini A, Matheus SC, Petroski EL. Imagem corporal em adolescentes: associação com a maturação sexual e sintomas de transtornos alimentares. J Bras Psiquiatr. 2010;59:198-202.

16. Dutra CL, Araújo CL, Bertoldi AD. Prevalência de sobrepeso em adolescentes: um estudo de base populacional em uma cidade no Sul do Brasil. Cad Saude Publica. 2006;22:151-62.

17. Madruga SW, Azevedo MR, Araújo CL, Menezes AMB, Hallal PC. Factors associated with weight loss dieting among adolescents: the 11-year follow-up of the 1993 Pelotas (Brazil) birth cohort study. Cad Saude Publica. 2010;26:1912-20.

18. Novaes JF, Franceschini SCC, Priore S. Hábitos alimentares de crianças eutróficas e com sobrepeso em Viçosa, Minas Gerais, Brasil. Rev Nutr. 2007;20:633-42.

19. Pinheiro AP, Giugliani ERJ. Body dissatisfaction in Brazilian schoolchildren: prevalence and associated factors. Rev Saude Publica. 2006:40:489-96.

20. Triches RM, Giugliani ERJ. Insatisfação corporal em escolares de dois municípios da região Sul do Brasil. Rev Nutr. 2007;20:119-28.

21. Fidelix YL, Silva DAS, Pelegrini A, Silva AF, Petroski EL. Insatisfação com a imagem corporal em adolescentes de uma cidade de pequeno porte: associação com sexo, idade e zona de domicílio. Rev Bras Cineantropom Desempenho Hum. 2011;13:202-7.

22. Martins CR, Pelegrini A, Matheus SC, Petroski EL. Insatisfação com a imagem corporal e relação com estado nutricional, adiposidade corporal e sintomas de anorexia e bulimia em adolescentes. Rev Psiquiatr Rio Gd Sul. 2010;32:19-23

23. Vale AMO, Kerr LARS, Bosi MLM. Comportamentos de risco para transtornos do comportamento alimentar entre adolescentes do sexo feminino de diferentes estratos sociais do Nordeste do Brasil. Ciênc Saúde Coletiva. 2011;16:121-32.

24. Antonaccio CMA. Estudantes de nutrição: uma ótica sobre o comportamento alimentar e os transtornos alimentares [dissertação]. São Paulo: FCF-FEA-FSP/USP; 2001.

25. Fiates GMR, Salles RK. Fatores de risco para o desenvolvimento de distúrbios alimentares: um estudo em universitárias. Rev Nutr. 2001;14:3-6.

26. Souza FGM, Martins MCR, Monteiro FCC, Neto GCM, Ribeiro IB. Anorexia e bulimia nervosa em alunas da Faculdade de Medicina da Universidade Federal do Ceará. Rev Psiq Clín. 2002;24:172-80.

27. Luz SS. Avaliação de sintomas de transtornos alimentares em universitárias de Belo Horizonte [tese]. São Paulo: Universidade de São Paulo; 2003.

28. Bosi MLM, Luiz RR, Morgado CMC, Costa MLS, Carvalho RJ. Comportamentos de risco para transtornos do comportamento alimentar e fatores associados entre estudantes de nutrição do município do Rio de Janeiro. J Bras Psiquiatr. 2006;55:34-40.

29. Feio LC. Prevalência de sintomas de anorexia nervosa e insatisfação com a imagem corporal em universitárias ingressantes na Universidade Federal de Santa Catarina [dissertação]. Florianópolis: Universidade Federal de Santa Catarina; 2007.

30. Bosi MLM, Luiz RR, Uchimura KY, Oliveira FP. Comportamento alimentar e imagem corporal entre estudantes de educação física. J Bras Psiquiatr. 2008;57:28-33.

31. Bosi MLM, Uchimura KY, Raggio Luiz R. Eating behavior and body image among psychology students. J Bras Psiquiatr. 2009;58:150-5.

32. Kirsten VR, Fratton F, Porta NBD. Transtornos alimentares em alunas de nutrição do Rio Grande do Sul. Rev Nutr. 2009;22:219-27.

33. Laus MF, Moreira RCM, Costa TMB. Diferenças na percepção da imagem corporal, no comportamento alimentar e no estado nutricional de universitárias das áreas de saúde e humanas. Rev Psiquiatr Rio Gd Sul. 2009;31:192-6.

34. Alvarenga M, Scagliusi FB, Philippi ST. Comportamento de risco para transtorno alimentar em universitárias brasileiras. Rev Psiq Clín. 2011;38:3-7.
35. Pereira LNG, Trevisol FS, Quevedo J, Jornada LK. Eating disorders among health science students at a university in southern Brazil. Rev Psiquiatr Rio Gd Sul. 2011;33:14-9.

36. Coqueiro RS, Petroski EL, Pelegrini A, Barbosa AR. Insatisfação com a imagem corporal: avaliação comparativa da associação com estado nutricional em universitários. Rev Psiquiatr Rio Gd Sul. 2008;30:31-8.

37. Alvarenga M, Philippi ST, Lourenco BH, Sato PM, Scagliusi FB. Insatisfação com a imagem corporal em universitárias brasileiras. J Bras Psiquiatr. 2010;59:44-51.

38. Vieira JLL, Amorim HZ, Vieira LF, Amorim AC, Rocha PGM. Distúrbios de atitudes alimentares e distorção da imagem corporal no contexto competitivo da ginástica rítmica. Rev Bras Med Esporte. 2009;15:410-4.

39. Ribeiro $L G$, Veiga GV. Imagem corporal e comportamentos de risco para transtornos alimentares em bailarinos profissionais. Rev Bras Med Esporte. 2010;16:99-102.

40. Cobelo AW, De Chermont PEC, Nakano EY, Conti MA, Cordás TA. Body image dissatisfaction and eating symptoms in mothers of adolescents with eating disorders. Eat Weight Disord. 2010;15:e219-25.

41. Soares RM, Nunes MA, Schmidt MI, Giacomello A, Manzolli P, Camey S, et al. Inappropriate eating behaviors during pregnancy: prevalence and associated factors among pregnant women attending primary care in southern Brazil. Int J Eat Disord. 2009:42:387-93.

42. Silva DA, Nahas MV, De Sousa TF, Del Duca GF, Peres KG. Prevalence and associated factors with body image dissatisfaction among adults in Southern Brazil: a population-based study. Body Image. 2011;8:427-31.

43. Perini TARS, Vieira RS, Vigário OS, Oliveira GL, Ornellas JS, Oliveira FP. Transtorno do comportamento alimentar em atletas de elite de nado sincronizado. Rev Bras Med Esporte. 2009;15:54-7

44. Coutinho DC, Sichieri R, Benício MHA. Obesity and weight change related to parity and breast-feeding among parous women in Brazil. Public Health Nutr. 2001;4:865-70.

45. Haines J, Neumark-Sztainer D. Prevention of obesity and eating disorders: a consideration of shared risk factors. Health Educ Res. 2006;21:770-82.

46. Garner DM, Olmested MP, Bohr Y, Garfinkel PE. The eating attitude test: psychometric features and clinical correlates. Psychol Med. 1982;12:871-9.

47. Bighetti F, Santos CB, Santos JE, Ribeiro RPP. Tradução e validação do Eating Attitudes Test em adolescentes do sexo feminino de Ribeirão Preto-SP. J Bras Psiquiatr. 2004:53:339-46.

48. Stunkard A, Sorensen T, Schlusinger F. Use of Danish adoption register for the study of obesity and thinness. In: Kety S, Rowland LP, Sidman RL, Matthysse SW. The genetics of neurological and psychiatric disorders. New York: Raven; 1983. p. 115-20.

49. Scagliusi FB, Alvarenga M, Polacow VO, Cordás TA, Queiróz GKO, Coelho D, et al. Concurrent and discriminant validity of the Stunkard's Figure Rating Scale adapted into Portuguese. Appetite. 2006;47:77-82.

50. American Dietetic Association (ADA). Position of the American Dietetic Association: nutrition intervention in the treatment of anorexia nervosa, bulimia nervosa, and other eating disorders. J Am Diet Assoc. 2006;106:2073-82.

51. Slevec JH, Tiggemann M. Predictors of body dissatisfaction and disordered eating in middle-aged women. Clin Psychol Rev. 2011;31:515-24.

52. Hoek HW, Van Hoeken D. Review of the prevalence and incidence of eating disorders. Int $J$ Eat Disord. 2008;34:383-96.

53. Astrachan-Fletcher E, Veldhuis C, Lively N, Fowler C, Marcks B. The reciprocal effects of eating disorders and the postpartum period: a review of the literature and recommendations for clinical care. J Women Health. 2008;17:227-39.

54. Von Soest T, Wichstrøm $L$. The impact of becoming a mother on eating problems. Int J Eat Disord. 2008:41:215-23.

55. Paeratakul S, York-Crowe EE, Williamson DA, Ryan DH, Bray GA. Americans on diet: results from the 1994-1996 Continuing Survey of Food Intakes by Individuals. J Am Diet Assoc. 2002;102:1247-51.

56. Simon J, Schmidt U, Pilling S. The health service use and cost of eating disorders. Psychol Med. 2005:35:1543-51.

57. Benas JS, Uhrlass DJ, Gibb BE. Body dissatisfaction and weight-related teasing: a model of cognitive vulnerability to depression among women. J Behav Ther Exp Psychiatry. 2010:41:352-6. 
58. Polivy J, Herman CP. An evolutionary perspective on dieting. Appetite. 2006;47:30-5.

59. Vester K. Regime change: gender, class, and the invention of dieting in post-bellum America. J Soc Hist. 2010;44:39-70.

60. Nunes MA, Camey S, Olinto MTA, Mari JJ. The validity and 4-year test-retest reliability of the Brazilian version of the Eating Attitudes Test-26. Braz J Med Biol Res. 2005;38:1655-62.
61. Gardner RM, Friedman BN, Jackson NA. Methodological concerns when using silhouettes to measure body image. Percept Mot Skills. 1998;86:387-95.

62. Ferreira VA, Magalhães R. Obesidade e pobreza: 0 aparente paradoxo. Um estudo com muIheres da Favela da Rocinha, Rio de Janeiro, Brasil. Cad Saude Publica. 2005;21:1792-800. 